\title{
Preferred Primary Healthcare Provider Choice Among Insured Persons in Ashanti Region, Ghana
}

\author{
Micheal Kofi Boachie*
}

\begin{abstract}
Background: In early 2012, National Health Insurance Scheme (NHIS) members in Ashanti Region were allowed to choose their own primary healthcare providers. This paper investigates the factors that enrolees in the Ashanti Region considered in choosing preferred primary healthcare providers (PPPs) and direction of association of such factors with the choice of PPP.

Methods: Using a cross-sectional study design, the study sampled 600 NHIS enrolees in Kumasi Metro area and Kwabre East district. The sampling methods were a combination of simple random and systematic sampling techniques at different stages. Descriptive statistics were used to analyse demographic information and the criteria for selecting PPP. Multinomial logistic regression technique was used to ascertain the direction of association of the factors and the choice of PPP using mission PPPs as the base outcome.

Results: Out of the 600 questionnaires administered, 496 were retained for further analysis. The results show that availability of essential drugs $(53.63 \%)$ and doctors $(39.92 \%)$, distance or proximity $(49.60 \%)$, provider reputation (39.52\%), waiting time (39.92), additional charges (37.10\%), and recommendations (48.79\%) were the main criteria adopted by enrolees in selecting PPPs. In the regression, income (-0.0027), availability of doctors (-1.82), additional charges (-2.14) and reputation (-2.09) were statistically significant at $1 \%$ in influencing the choice of government PPPs. On the part of private PPPs, availability of drugs (2.59), waiting time (1.45), residence (-2.62), gender (-2.89), and reputation $(-2.69)$ were statistically significant at $1 \%$ level. Presence of additional charges $(-1.29)$ was statistically significant at $5 \%$ level.

Conclusion: Enrolees select their PPPs based on such factors as availability of doctors and essential drugs, reputation, waiting time, income, and their residence. Based on these findings, there is the need for healthcare providers to improve on their quality levels by ensuring constant availability of essential drugs, doctors, and shorter waiting time. However, individual enrolees may value each criterion differently. Thus, not all enrolees may be motivated by same concerns. This requires providers to be circumspect regarding the factors that may attract enrolees. The National Health Insurance Authority (NHIA) should also ensure timely release of funds to help providers procure the necessary medical supplies to ensure quality service.

Keywords: Ghana, Insurance, Healthcare Provider, Patient Choice, Multinomial Logit

Copyright: (c) 2016 by Kerman University of Medical Sciences

Citation: Boachie MK. Preferred primary healthcare provider choice among insured persons in Ashanti Region, Ghana. Int J Health Policy Manag. 2016;5(3):155-163. doi:10.15171/ijhpm.2015.191
\end{abstract}

\section{Article History:}

Received: 19 April 2015

Accepted: 16 October 2015 ePublished: 20 October 2015

*Correspondence to:

Micheal Kofi Boachie

Email: mkboachie@gmail.com

\section{Key Messages}

Implications for policy makers

- Enrolees choose their preferred primary healthcare providers (PPPs) based on their own personal characteristics and provider characteristics. However, different enrolees may have different motivations for selecting a particular PPP at a time.

- Policy-makers should allocate more funds to increase the number of primary healthcare facilities, especially in rural areas.

- The study also provides avenues for healthcare providers to optimise their roles in the health sector by improving quality levels. The National Health Insurance Authority (NHIA) should ensure timely disbursement of funds to providers to help them procure the necessary medical supplies.

Implications for public

The policy suggestions in the study will help improve satisfaction among patients. In this regard, enrolees should also make it a point to find more information about the providers they wish to choose as their preferred primary healthcare provider (PPP) and also switch providers when quality, in their perspective, is compromised to help ensure competition for quality.

\section{Background}

Across the globe, contemporary healthcare systems rely on competition as one of the mechanisms to contain the escalating healthcare costs. As pointed out by Annemarie and Hester, ${ }^{1}$ consumer choice is a pre-condition for competition to thrive in every market; and for decades, in health economics literature, patient (consumer) choice of healthcare provider has been seriously debated. Appleby et $\mathrm{al}^{2}$ have argued that patients will benefit, regardless of the choice they make, when offered a choice; and will consequently augment the cost 
containment strategies of purchasers.

In recent years, the patient choice concept has attracted considerable attention, for instance in the Netherlands, the United States, and the United Kingdom, due to its potential to create a more patient-centred care, improve efficiency, quality, and equity in healthcare use..$^{3-5}$ In most advanced economies, with well-developed healthcare markets, health insurers and patients have the freedom to choose which hospital to contract and/or visit with fewer complexities.

One of the myriad ways of offering patients a choice is through patient list systems (as happens in Norway, the United States, and Denmark). Generally, patient list systems allow enrolees to choose their own healthcare providers or general practitioners (GPs) where they would receive treatment in times of sickness or emergency cases such as motor accidents. Enrolees have the opportunity to change providers, after a certain period, if they are not satisfied with service delivery. Consequently, patients may substitute distant healthcare providers for nearer ones due to differences in quality care, waiting times, and hospital reputation. ${ }^{6-9}$ In certain cases, purchasers (insurers) have limited and/or specified healthcare providers from which their patients can seek treatment, and usually, purchasers' (insurers) bargaining power partly depends on patients' willingness to change provider. ${ }^{5}$

In early 2012, Ghana's National Health Insurance Authority (NHIA) offered its enrolees in the Ashanti Region the option to choose their own primary healthcare providers via a patient list system called preferred primary healthcare provider (PPP). It was the NHIA's intention to extend, after a year, the practice to the entire country depending on the success of the pilot in Ashanti Region. The nationwide implementation of the patient list system was expected to take-off after December 2012. In view of this, NHIA accredited healthcare providers operating in the Ashanti Region (were) are usually prepaid for future provision of defined services to NHIA patients on the provider's register. Enrolees are (were) at liberty to switch from one healthcare provider to the other, once in every 6 months, if they are not satisfied with services received from the PPP.

In effect, the revenue of healthcare providers, especially private healthcare providers, would depend, partly, on the number of National Health Insurance Scheme (NHIS) subscribers on their roll. This has the potential to spark competition for quality healthcare delivery in Ghana. Indeed, this was one of NHIA's motivations for the patient list system among others like improving pricing and reimbursement activities, and efficiency of providers. ${ }^{10}$ The success or otherwise of the programme and/or its effect on patients is not within the purview of the present study. ${ }^{11-14}$

The basis for patient choice is rooted in Hirschman's ${ }^{15}$ principle of 'voting your feet.' The general idea is that consumers have the option to withdraw or complain when there is a decline in the quality of service and/or benefit. Thus, those who are unhappy with their organization may exit to another organization for a better service. By withdrawing, consumers (eg, enrolees) substitute non-performing providers for performing ones. This substitution of providers will require consumers (enrolees) desiring high-quality care at minimal costs to acquire information regarding prices and quality of different healthcare providers to make an informed decision as to which provider best meets their preferences and needs. Every insured member, based on his/her own information, chooses (and sometimes changes) PPP in this system. Consequently, examining the factors that influence patients' choice of PPP could be important towards ensuring quality in healthcare delivery via patient choice. Our objective in this paper is to investigate the factors that affect the choice of PPP, and their direction of association and magnitude with the choice of PPP. Indeed, some studies on patient hospital (provider) choice and/or utilisation of healthcare services have been conducted in developing countries ${ }^{16-20}$ and developed countries. ${ }^{1,6,8,9,21-24}$ However, most of these studies, eg, Dzator and Asafu-Adjaye, ${ }^{17}$ examined patient hospital choice under conditions where price (user fees) existed and/ or for a particular disease. Similar to Varkevisser et al, ${ }^{9}$ the present study focuses on factors that affect the choice of PPP in the absence of user fees for insured persons.

\section{Methods \\ Study Design}

The study used a cross-sectional research design. Thus, a short community-based survey was used to collect data at a specific point in time, without any follow-up. Questionnaires were used to elicit information from respondents. Generally, the cross-sectional study design allows different variables to be compared simultaneously hence our choice of this approach.

\section{Setting and Population}

The study was conducted in the Ashanti Region, which is located in the middle belt of Ghana. The region has 4780380 inhabitants representing $19.4 \%$ of Ghana's entire population ${ }^{25}$ with a mixture of rural and urban communities as well as a representative collection of small, medium, and big healthcare facilities. Administratively, the region is divided into 27 districts as at 2010 to facilitate the decentralisation process. Currently, the NHIA is practicing the patient list system (PPP) in the region. Since the objective of this study is to learn how enrolees choose their PPPs, the choice of Ashanti Region for this study is justified. This is because the patient list system was not available in any other region. For the purposes of this study, the population comprised of NHIS enrolees in the region. ${ }^{26}$ Eligible participants were insured persons of age 18 or above who had selected and/or changed their PPP within 6 months prior to the survey. The study relied solely on primary data.

\section{Sample, Sampling Procedures, and Data Collection}

Yamane's ${ }^{27}$ [1] sample size formula was applied and the minimum sample size was 400 NHIS enrolees using 95\% confidence and $5 \%$ error levels. In order to account for damages, incomplete responses, missing questionnaires and other problems associated with data collection, 50\% extra of the sample size was allowed to address such problems. It is acknowledged that in most empirical surveys $10 \%$ of the estimated sample size has been applied to address data collection problems. The choice of $50 \%$ extra in the present study, however, was to help achieve a relatively larger sample size for greater efficiency in the multinomial logit estimation. The sample size was distributed between rural and urban districts in the region based on their respective total 
populations. Prior to this, a list of rural and urban districts ${ }^{[2]}$ in the region was made separately after which a simple random sampling procedure was applied to select one district from each list. In the process, each of the districts was given a number based on which the selection was made. Kumasi Metro area (urban) and Kwabre East (rural) were the 2 districts selected for the study. In each of the selected districts was applied a simple random and systematic sampling procedures at different stages.

First, in Kumasi Metro, one submetro (ie, Oforikrom) was selected out of the 10 submetros after which systematic sampling method was used to select houses to visit in order to interview 400 individual enrolees ${ }^{[3]}$. The selection of Oforikrom submetro was based on a list that contained all the ten submetros with their assigned numbers. Secondly, in Kwabre East district, a list of all the communities was made and numbers were assigned based on which the selection of 2 villages (ie, Kasaam and Adwumakase-Kese) was done without replacement ${ }^{[4]}$. Thus, once the first village is selected it is not replaced. This reduces the total number of villages in the next selection. The 2 villages were allotted 100 questionnaires each totalling 200. In all cases, the listing and numbering were done by the researcher(s).

There was no listing of houses. The first house was picked and subsequently every fifth house was visited. If the subsequent house has no eligible member, the next house was visited in order to get eligible participant. This procedure was followed until the required sample size was achieved. Once in the house, anybody (male or female) present could answer the questions posed by the interviewer (or if literate select their answers from the questionnaire), provided he/she satisfies the eligibility criteria. Only insured persons who had attained age 18 or older and had selected and/or changed PPP within 6 months prior to the study were eligible. Selection of a participant in the house was conveniently done.

Prior to the interview/survey, author-developed research questionnaire was first administered to 5 insured persons, residents of Kumasi, who had selected their PPPs. However, this selection was unscientific. The returned questionnaires suggested the need for revision as there was duplication of questions while some questions needed multiple responses. The revised questionnaire was then administered to eligible participants after seeking their verbal consent.

The questionnaire was designed in way that would help capture, for example, information on socio-demographic characteristics (eg, gender, age, and income), factors that were considered in choosing PPP, whether or not participants have switched provider, distance to PPP captured by travel time, and transportation cost. Thus, the questionnaire was in 2 sections. Section A comprised of questions on individual or personal characteristics (eg, gender, age, and income). In section $B$, the questions were related to, for example, the type of PPP chosen, why that PPP was chosen, and location of the chosen PPP.

Predictive Model for Choice of Preferred Primary Healthcare Provider

In most of the relevant literature, decisions about hospital choice among patients are discrete. Therefore, they can be modelled quantitatively. Likewise, decisions regarding the choice of PPP can also be modelled quantitatively. In line with such authors as Amaghionyeodiwe, ${ }^{18}$ and Chul-Young and Keon-Hyung ${ }^{24}$ enrolees were assumed to choose their PPP from among Government, Private, and Mission healthcare providers (either in their locality or bypassing the nearest healthcare provider to a more distant one). The type of provider served as the dependent variable. Thus, each enrolee chooses his/her PPP on the premise that he/she derives maximum utility from treatment at the chosen facility. The individual's conditional expected utility function is given as:

$U_{i j}=V_{i j}\left(D_{i}, H_{j}\right)$

Where $U_{i j}$ is the individual's expected utility conditional on receiving treatment from PPP (provider) $j, V_{i j}\left(H_{j}, D_{i}\right)$ is the observed utility; $D_{j}$ is a vector of individual characteristics such as age, gender, income, education, and location; $H_{j}$ is a vector of PPP characteristics such as drug availability, doctors, and proximity of the facility to the individual. Hence, an enrolee's conditional expected utility can be given as:

$U_{i j}=V_{i j}\left(P P P / D_{i}, H_{j}\right)$

In effect, the enrolee chooses his PPP given his/her own characteristics, disease (illness) features, and that of the healthcare provider to maximise his/her utility from treatment. Thus, the enrolee chooses his/her PPP given his/ her income, gender, education, and facility features such as drug availability, charges aside insurance, and availability of healthcare personnel. The reduced form of the utility function is presented below:

$U_{i j}=V_{i j}\left(P P P / D_{i}, H_{j}\right)=\alpha+\beta_{1} D_{i}+\beta_{2} H_{j}+\delta_{i}$

where all notations are as previously defined, and the error term is represented by $\delta_{i}$. Here the enrolee chooses provider or facility $j$ if and only if it offers him/her the highest utility. In general, the probability of an enrolee choosing provider $j$ as PPP given facility and individual features is given by:

$\pi_{i j}=\frac{\delta^{\alpha_{j}+\beta_{j} X}}{\sum \delta^{\alpha_{j}+\beta_{j} X}}$

Where $\pi_{i j}$ is the probability that enrolee $i$ would select healthcare provider $j$ as his/her PPP, $\delta$ is the error term, $\alpha_{j}$ is the intercept while $\beta_{j}$ is the vector coefficients of the independent variables $(X)$ and $X$ is a vector of variables affecting the choice of healthcare provider as detailed above. In order to measure the direction of association of the factors with choice of PPP, the study makes use of multinomial logistic regression. The choice of multinomial logistic regression stems from the multicategory (dissimilar) nature of the options available to the enrolee, and it is consistent with studies modelling choice behaviour. ${ }^{16-18}$

Since an enrolee chooses a PPP irrespective of sickness, this study excludes disease specific features of the individual. Thus, we make use of individual characteristics such as gender, education, age, income, residence, and facility features such as drug availability, waiting time and other factors such as distance between the individual's residence and the facility and travel time. These served as independent variables in the multinomial logistic regression. While patients' characteristics are very influential in selecting a particular healthcare provider, the characteristics of the provider are equally important. This is because the enrolee 
might choose a particular healthcare provider mainly due to certain characteristics that the provider possesses.

\section{Data Analysis}

The data was first entered in Microsoft Excel, and verified before transferring same to Stata 11. Both descriptive and inferential statistics were used to analyse the data. The differences in responses and background information were obtained using percentages, averages and standard deviations. A multinomial logistic regression was used to ascertain the direction of associations between the independent variables and choice of PPP, and their magnitudes. All categorical independent variables entered the regression equation in the form of indicator variables, taking the values of zero and one $(0$ and 1$)$ accordingly. For statistical significance, $P$ value of .05 or less was applied.

\section{Results}

Data Description

Overall, 600 participants were recruited for the study. However, 496 questionnaires were retained for further analysis after accounting for incomplete, damaged, and missing questionnaires. This gave a response rate of nearly $83 \%$ and was satisfactory. Most of the respondents recruited for the study came from urban centres following the large number of residents in these areas, and constituted $68.15 \%$ of the sample. The rural respondents were $31.85 \%$ of the sample. The average age of a respondent was 35.65 years $(\mathrm{SD}=13.64)$ and the average monthly income was GH\$579.65. The mean travel time for a respondent to visit his/her PPP was 55 minutes and 38 seconds ( $\mathrm{SD}=36$ minutes) while the average transportation cost was $\mathrm{GH} 49.65(\mathrm{SD}=5.92)$. Females constituted a larger share of the respondents (see Table 1). Again, $80.65 \%$ of the respondents were employed and married people constituted $64.52 \%$ of the sample. Regarding educational background, only $5.65 \%$ of the respondents had no formal education. For those with formal education, $12.10 \%$ of the respondents had completed basic school (ie, junior secondary/high school) as at the time of the study. Post-basic school leavers from senior secondary/high, technical and/or vocational schools constituted $12.10 \%$ of the sample. Finally, $70.16 \%$ of the respondents had attained tertiary education (ie, post-secondary school education eg, polytechnic, university, college of education, and/or nursing). The socio-demographic characteristics of respondents such as employment and marital status, and their travel time and transport cost are presented in Table 1.

\section{Criteria for Selecting Preferred Primary Healthcare Provider} Among Enrolees

Majority of the respondents chose government health facilities as their PPPs. This represented $59.48 \%$ of the total sample. Enrolees who registered with private healthcare providers in the region constituted $33.27 \%$ of the respondents while mission healthcare providers accounted for $7.26 \%$ of the sample. Most (73.39\%) of the PPPs chosen were located within Kumasi metropolis.

Concerning the criteria for selecting PPPs, the respondents indicated that proximity, shorter waiting times, availability of essential drugs, and doctors were among the criteria used.
Table 1. Socio-Demographic Characteristics of Respondents

\begin{tabular}{|c|c|}
\hline Variable & Proportion \\
\hline \multicolumn{2}{|l|}{ Residence (\%) } \\
\hline Rural & 31.85 \\
\hline Urban & 68.15 \\
\hline \multicolumn{2}{|l|}{ Gender (\%) } \\
\hline Male & 48.39 \\
\hline Female & 51.61 \\
\hline \multicolumn{2}{|l|}{ Employment (\%) } \\
\hline Employed & 80.65 \\
\hline Unemployed & 19.35 \\
\hline \multicolumn{2}{|l|}{ Education (\%) } \\
\hline Basic (Junior secondary/high School) & 12.10 \\
\hline Secondary/technical/vocational & 12.10 \\
\hline Tertiary (Post secondary/technical/vocational) & 70.16 \\
\hline Uneducated & 5.65 \\
\hline \multicolumn{2}{|l|}{ Marital status } \\
\hline Married & 64.52 \\
\hline Single & 35.48 \\
\hline Mean income (in Ghana cedis ${ }^{a}$ ) & 579.65 \\
\hline (SD) & $(346.75)$ \\
\hline Mean Age (in completed years) & 35.65 \\
\hline (SD) & $(13.64)$ \\
\hline Mean travel time (in minutes) & 55.38 \\
\hline (SD) & (36.28) \\
\hline Mean transport cost (in Ghana cedis) & 9.65 \\
\hline (SD) & $(5.92)$ \\
\hline
\end{tabular}

a US\$1 = GHC3.8.

Table 2. Criteria for PPP Selection in Ashanti Region

\begin{tabular}{lc}
\hline Criteria & $\begin{array}{c}\text { Proportion of } \\
\text { Respondents (\%) }\end{array}$ \\
\hline Availability of drugs & 53.63 \\
Shorter waiting time & 39.92 \\
Proximity & 49.60 \\
Clean environment & 39.52 \\
Availability of doctors & 39.92 \\
Presence of additional charges aside insurance & 37.10 \\
Reputation of the provider & 39.52 \\
Recommendation from friends, co-workers, etc & 48.79 \\
\hline
\end{tabular}

Abbreviation: PPP, preferred primary healthcare provider.

Table 2 summarizes the criteria for selecting a PPP in Ashanti Region among enrolees.

It is evident from Table 2 that more than half of NHIS enrolees selected their PPPs based on availability of essential drugs. Such information was made known via their past visits and recommendation. Another important criterion was distance. A percentage (49.60\%) of the enrolees preferred to be treated in a nearby health facility or hospital. Again, recommendations were also key in PPP selection as $48.79 \%$ based their selection on recommendation from friends, coworkers, and family members. Further, averagely 39\% of the sample included reputation of the provider, availability of doctors, clean environment, and shorter waiting times in the selection criteria. None of the enrolees switched provider within 6 months prior to the study.

Estimates From Multinomial Logistic Regression for Choice of Preferred Primary Healthcare Provider

The full maximum likelihood estimation method was used 
to fit the multinomial logistic regression. Our objective here was to measure the extent of association of some the factors (see Table 2) or their influence on enrolees' decision to choose a particular PPP. As explained earlier, the choice of PPP (government, private or mission) was dependent on both individual and facility characteristics. The results from the multinomial logistic regression are summarized in Table 3. The overall regression estimates are satisfactory. The likelihood ratio chi-square was statistically significant suggesting that at least one of the regression coefficients was statistically different from zero. The pseudo $\mathrm{R}^{2}$ was approximately 0.37 implying that about $37 \%$ of the changes in the dependent variable are explained by the independent variables presented in the model. These estimated coefficients indicate how the factors influence the likelihood of an enrolee selecting a particular PPP. The negative sign of an estimated coefficient indicates a lesser chance or likelihood while a positive sign of the coefficient indicates a greater chance of selection.

Gender was significant in deciding whether to join private PPP. However, it was statistically insignificant in determining the decision to select a government owned facility as PPP. The coefficient of gender was negative for both government and private providers. In addition, enrolee's age was positively related to the demand for healthcare services from both public and private providers. It was, however, statistically significant at $10 \%$ for only private providers.

The level of an enrolee's education was not significant in determining his/her choice of PPP in Ashanti Region. Surprisingly, the coefficients were negative for both public and private healthcare providers at all levels of education. Further, income was negatively related to the choice of government providers at $1 \%$ and private providers at $10 \%$. Again, the effect of enrolees' residence was statistically

Table 3. Multinomial Logistic Estimates for Choice of PPP

\begin{tabular}{lcc}
\hline Variable & Government Providers & Private Providers \\
\cline { 2 - 3 } & Coefficients & Coefficients \\
\hline Enrolee characteristics & & \\
Gender: male & -0.3527 & $-2.8886^{\mathrm{a}}$ \\
Age & 0.0264 & $0.0328^{\mathrm{b}}$ \\
Basic education & -19.6099 & -19.1138 \\
Secondary education & -16.9830 & -14.2808 \\
Tertiary education & -16.8314 & -16.7729 \\
Income & $-0.0027^{\mathrm{a}}$ & $-0.0010^{\mathrm{b}}$ \\
Residence-rural & $-0.9321^{\mathrm{b}}$ & $-2.6162^{\mathrm{a}}$ \\
Facility features & & \\
Availability of drugs & 1.2253 & $2.5911^{\mathrm{a}}$ \\
Shorter waiting time & -0.1320 & $1.4486^{\mathrm{a}}$ \\
Clean environment & 0.1208 & $-1.1775^{\mathrm{b}}$ \\
Availability of Doctors & $-1.8210^{\mathrm{a}}$ & $1.9270^{\mathrm{a}}$ \\
Presence of additional & $-2.1442^{\mathrm{a}}$ & $-1.2934^{\mathrm{c}}$ \\
charges aside insurance & $-2.0851^{\mathrm{a}}$ & $-2.6946^{\mathrm{a}}$ \\
Reputation of the provider & -0.0005 & 0.0005 \\
Distance (travel time) & 23.1486 & 19.6297 \\
Constant & & \\
\hline
\end{tabular}

Abbreviation: PPP, preferred primary healthcare provider.

a, b, c Denotes significance at $1 \% .10 \%$, and $5 \%$ level, respectively.

Summary Statistics: Obs, 496; R2-Pseudo, 0.3705; Log-likelihood, 270.2479;

LR chi-square (28), 318.1400; $P$ value: .0000 .

Base outcome is mission health providers.

Results obtained from STATA version 11. insignificant for government providers. Precisely, the sign of the coefficient of residing in a rural community was negative for both government and private providers. However, it was significant at $1 \%$ for private providers while the negative effect was insignificant at $5 \%$ for government healthcare providers. Facility features such as availability of drugs and doctors, shorter waiting times, clean environment, provider's reputation, and presence of additional charges were found to exert significant influence on an enrolee's decision to select a particular provider as PPP within the sample. The availability of essential drugs was a positive determinant of PPP. While this was statistically significant at $1 \%$ for private healthcare providers, it was insignificant for government PPPs.

Shorter waiting time was a positive determinant of private healthcare providers' services while it was negative for public providers, and it was statistically significant at $1 \%$ for private providers. Furthermore, clean environment was positively related to the choice of government PPP though insignificant while it related negatively to the choice of private providers and statistically insignificant at 5\% level. The availability of medical doctors in a particular facility was found to be very influential in selecting PPPs among enrolees. This criterion was statistically significant at $1 \%$ for both government and private providers in the region though they moved in opposite directions. Specifically, availability of doctors was positive for private providers while it remained negative for public providers.

The presence of additional charges in a facility and reputation of the provider were negative in terms of their influence on enrolees' decision to choose a particular facility as PPP. As shown in Table 3, its effect was negative for both government and private providers. The coefficient of providers' reputation was negative and statistically significant at $1 \%$ for both government and private providers. Distance (measured in travel time) was, however, not a significant determinant of PPP choice among enrolees. However, its sign was negative for public while it remained positive for private providers.

\section{Discussion}

In the present study, 496 respondents have provided information on the factors they considered/consider when choosing a PPP. Our results suggest a large number of female subscribers of the health insurance policy. This high percentage of females in the present study could emanate from their frequent use of healthcare services and thus their interest in subscribing to the health insurance policy. Another possibility for this high percentage is the absence of men in the house as at the time of interview. However, we consider the difference in male and female percentages to be marginal since it is below $10 \%$.

The findings on employment and education are interesting. We find that $80.65 \%$ of the respondents were employed as at the time of the study. A possible reason for this percentage is that there were only 2 options regarding employment status and as such those who did not find their category might have selected "employed" option. For example, the questionnaire did not have any option for students and this could account for the high percentage. Further, there was no categorisation of employment, which is a shortfall of the present study. Similarly, the demographic information reveals a high 
percentage of people with tertiary education and low percentage with basic or secondary education. Perhaps, a significant portion of these people was still in school, which our questionnaire did not capture. Indeed, the absence of some options on the questionnaire might have accounted for this. Another finding about the sample is on income. We find that the average income in the sample was GH\$ 579.65. This high average income could be due to some outliers in the sample that might have accounted for the large variation in income.

The study has shown that most of the PPPs selected are located in the Kumasi metropolis, and most of them are public facilities. We do not find this to be surprising given the large number of residents. Again, the high level of infrastructural development and other facilities in the metropolis make it conducive for entities such as hospitals to be sited. The higher preference for government health providers stems, partly, from the withdrawal of services by private healthcare providers on grounds of low capitated rates in the early stages of the PPP.

The findings regarding continuity of care contradicts Boachie's ${ }^{11}$ findings that enrolees in the patient list system had a higher probability of switching providers during the piloting stage of the programme. Indeed, the early stages of the patient list system witnessed some hitches. Most patients were turned away by providers, particularly private providers, on grounds that those patients were not on their register. This problem was due to the random assignment of enrolees to providers without the knowledge of concerned enrolees. ${ }^{11}$ However, such issues were later addressed. This could explain the results in the present study.

Intuitively, the insured have their own guidelines in selecting a PPP when given a choice. We find that enrolees selected their PPPs based on perceived availability of drugs and doctors in such facilities. Thus, enrolees chose facilities that have high probability of consulting a doctor, and also receive essential drugs. Usually, this information is made known through past visits or from friends/family members. Another criteria adopted was distance or proximity to the facility. Proximity to the health facility is indicative of lower transport cost (in both money and non-monetary terms) thus inducing enrolees to choose closer facilities. This could be the reason for the present results. In Nigeria, Amaghionyeodiwe ${ }^{18}$ has reported that $33 \%$ of respondents changed healthcare provider due to proximity. Similar to the findings reported by Schwartz et $\mathrm{al}^{6}$ among elderly people in the United States, recommendations were important criteria used by enrolees in selecting PPPs. In addition, waiting time was one of the criteria used in selecting PPPs. Waiting time in health facilities is one of the non-pecuniary costs to the patient. Undeniably, patients prefer to spend less time in health facilities hence 39.92\% of respondents using this criterion. Again, we find that provider reputation, clean environment, additional charges and recommendation were key guidelines that were adopted by enrolees. The enrolee's past visits and recommendations from friends, families, and co-workers could reveal essential information on, for instance, waiting time, sanitation, availability of drugs and doctors, and extra charges.

In examining the direction of association of some of these factors in the multinomial regression, we find that such individual specific characteristics as gender and age were significant in selecting PPP. Our results suggest that males gave less preference to private PPPs relative to females. We find this to be surprising. This is because males are mostly the breadwinners in traditional Ghanaian family settings, and as such will have a higher opportunity cost in seeking care. Given that private PPPs provide services within a short period, one would expect males to prefer such PPPs but our findings suggest otherwise. We are unable to find reasons for this behavior. This calls for further research in this area. In addition, each additional year increases the likelihood of an enrolee demanding the services of private healthcare providers. These findings are similar to those reported by Chul-Young and Keon-Hyung ${ }^{24}$ in the United States, and Amaghionyeodiwe $^{18}$ in Nigeria. However, the insignificant negative effect of education deviates absolutely from the findings of Amaghionyeodiwe. ${ }^{18}$

Further, income significantly influences the choice of PPPs. Specifically, increase in income reduces the likelihood of an enrolee selecting both government and private providers as PPP. However, the negative effect of rising income on private providers was less pronounced resulting, perhaps, from the shorter waiting time, and continuous availability of doctors to attend to patients. This resonates with the argument advanced by Amaghionyeodiwe ${ }^{18}$ that high-income earners perceive the quality of care in private health facilities to be higher with less time than in publicly owned facilities. In addition, Adaman et a ${ }^{19}$ have reported findings, similar to the present study, that the rich or people with high socio-economic status are more likely to choose private hospitals due to the shorter-waiting time and perceived quality. These could be the reason for the current results.

Again, residing in a rural community was negatively associated with selecting government or private providers, and is consistent with findings reported by Bour. ${ }^{20}$ However, this was statistically significant at conventional levels for only private PPPs.

The implication is that rural dwellers were less likely to select private providers as their PPP as compared with public and mission providers. This stems from the fact that most private health facilities are located in cities. In addition, most healthcare facilities in rural settings belong to either government or religious bodies thereby making it convenient for rural dwellers to utilise healthcare service in such facilities hence the insignificant effect of rural-residence on public PPPs. Again, the negative coefficient suggests some inequalities in healthcare use. Thus, between urban and rural areas, there exist some inequalities in accessing healthcare services, particularly services by private providers. There is an urgent need to address these issues of inequalities in healthcare utilisation.

Also, facility features such as availability of drugs and doctors, shorter waiting times, clean environment, provider's reputation, and presence of additional charges highly influence enrolees' decision to select a particular provider as PPP, or even healthcare provider in general. The enrolees expecting to receive essential drugs select private providers due to their continuous supply of such drugs. The implication is that enrolees who expect to receive essential drugs will select private providers as their PPP rather than government 
providers. This suggests that private providers supply essential drugs to their patients than public providers.

As already indicated, waiting time in health facilities is one of the non-pecuniary costs to the patient, and, in most cases, private providers are perceived to reduce this type of cost for patients. Our present results may partly emanate from the punctuality of doctors and other medical staff in private facilities. Another possible reason for the shorter waiting time in private facilities could be due to the fewer number of clients they serve since most people utilise public health facilities thereby causing less pressure on private facilities. The findings corroborate Varkevisser and van der Geest ${ }^{8}$ results concerning hospital bypassing, Amaghionyeodiwe's ${ }^{18}$ explanation for the choice of private hospitals among higher income groups in Nigeria, and Naasegnibe and Dary ${ }^{16}$ that time is a very important factor in deciding where to seek care in Northern Ghana.

The availability of doctors in a particular facility influences the selection of PPPs among enrolees. The results for government providers could be attributed to the dual practice by most of the medical doctors. Most private health facilities are owned, and (sometimes) operated by health professionals working in government facilities. Since revenue of private practice depends mostly on the number of patients, it becomes expedient for doctors to be punctual in such facilities and that could reduce waiting time in such private facilities. Usually, such doctors spend more time on their private practice than in the government facilities where remuneration is fixed. In some cases, supplier-induced demand in medical care is rampant among private practitioners. These, perhaps, are the reasons for the current results. These findings regarding provider characteristics confirm Varkevisser and van der Geest's $^{8,9}$ findings that hospital or facility features play a key role in hospital choice, or, in this case, PPP choice.

In choosing PPPs, enrolees were more likely to stay away from facilities that charge fees aside the insurance. The negative coefficients suggest that all the providers, whether government or private facilities, charged extra fees regardless of the insurance. The presence of additional fees in health facilities could be due to co-payments or payments for uncovered benefits under the insurance scheme though the study did not investigate the purpose for such fees. However, the effect was more pronounced among government providers than among private providers in the region as shown by their respective significance levels and coefficients. This additional fee charged could reduce utilisation of healthcare services, especially for the poor. Undoubtedly, user fee reduces utilisation of healthcare services in most cases and Boachie ${ }^{11}$ found that charging an extra fee aside insurance reduced visits among patients under fee-for-service and capitated plans. Provider reputation is among the factors influencing healthcare utilization decisions. Thus, providers who provide quality healthcare services in the form of shorter waiting time, provision of essential drugs to patients, clean environment, politeness of healthcare staff and other modern medical equipment would be expected to attract more patients. However, the negative and statistically significant coefficient of providers' reputation indicates that providers were less reputed. This could be due to the fact that some of the selected PPPs lacked some qualities such as modern medical equipment, shorter waiting time and/or essential drugs. The regression result on distance (measured in travel time) was statistically insignificant determinant of PPP choice among enrolees, despite $49.6 \%$ of respondents using this criterion. The supply of essential drugs, shorter waiting time, and perceived quality of care could render distance unimportant in selecting a particular PPP. Indeed, Montefiori, ${ }^{7}$ Varkevisser and van der Geest $^{8,9}$ have argued that [private] healthcare providers who provide compensation in the form of shorter waiting time, availability of essential drugs and doctors inter alia to patients are more likely to be chosen and that could render distance irrelevant for patients. In this case, patients may substitute distant healthcare providers for nearer ones due to differences in quality of care. This, perhaps, explains the insignificant coefficient of distance in the multinomial regression. In this study, the insignificant coefficient of distance deviates from that of Varkevisser and van der Geest, ${ }^{8,9}$ Naasegnibe and Dary, ${ }^{16}$ Amaghionyeodiwe, ${ }^{18}$ Chul-Young and Keon-Hyung ${ }^{24}$ and Bour ${ }^{20}$ who found distance to be a significant factor in healthcare provider choice.

\section{Conclusion}

In this study, the purpose was to investigate the factors that affect the choice of PPP and their direction of association with PPP. One advantage of patient choice and behaviour research is that it enables health insurers to know the individual, facility and other characteristics influencing the choice of PPP to aid their purchasing roles. It also helps hospital administrators to optimize their provision of healthcare services. Certain characteristics of the providers were used as a basis for PPP selection in the Ashanti Region. The study has revealed that shorter waiting time, availability of essential drugs and doctors, proximity, recommendations, clean environment and presence of additional charges were the criteria adopted by enrolees in choosing their PPPs.

These findings provide impetus for healthcare providers to improve on their quality levels by reducing waiting time, ensuring a constant supply of essential drugs, recruiting and making sure that doctors are at work, provide proper sanitation, and above all, remove additional charges that may restrict the insured from accessing healthcare.

More specifically, government healthcare providers should endeavour to ensure that medical doctors are always available to attend to patients while removing all charges (except eg, co-payment) for the insured. These factors were statistically significant. On the part of private providers, further reduction in waiting time via, for example, punctuality of medical staff, and removal of additional charges coupled with improved sanitation will not be a wasteful investment. Though not emanating directly from the study, NHIA should also ensure timely release of funds to providers to help procure the necessary medical supplies towards enhancing the work of both medical and nonmedical staff. This will improve service quality in the form of, for example, essential drugs, and clean environment.

Further, government should ensure adequate provision of infrastructure, particularly in rural areas to help reduce inequalities in healthcare use. The lack of adequate healthcare infrastructure in rural communities has been revealed by the negative coefficient of residing in rural areas. This limits 
healthcare utilisation among rural dwellers. In addressing such issues, while government expands the concept of community health planning services (CHPS) in rural areas, providing some incentives to private and mission providers to establish in rural areas will also help increase the number of primary healthcare facilities. This will require investments in such infrastructure as road networks, electricity, water supply, and telecommunications, which are lacking in rural areas. The investment in infrastructure, especially road networks, will provide easy means of transport to access health facilities in distant communities.

Admittedly, the profit maximization rule in economic theory, ie, marginal revenue equals marginal cost, makes the survival of private and/or mission primary healthcare providers in rural areas blur, given the low incomes in such areas. However, since the NHIA's capitated rate for primary healthcare is uniform, though varies among the three providers, irrespective of geographical location, it seems possible for private and/or mission providers to satisfy the profit maximization rule in rural areas regarding primary healthcare. Again, such private and/or mission providers operating in rural areas could be given top-up rate to cater for their location.

\section{Limitations of the Study}

This study is not without limitations. First, no qualitative assessment was done to find out the factors that influence PPP decisions. The qualitative approach could provide some detailed information about the decision-making process regarding "how and why" in PPP decisions among enrolees. In addition, the study did not elicit information from healthcare providers or PPPs and regulators, especially NHIA. Such information from providers and NHIA would have provided a better understanding of the patient list system and the choice process. Further, employment status and income of respondents were not categorized into groups. Such categorisation could also provide information on how different employment and income groups choose PPPs. Qualitative analysis on the theme could be done in the future while incorporating these concerns to understand, better, how and why enrolees make decisions regarding PPPs. Finally, our pre-testing of the questionnaire used a small number of people. This, perhaps, did not help to reveal all shortcomings of the questionnaire. Future pre-testing should use relatively more people to help reveal the shortcomings in the research instrument.

\section{Acknowledgements}

I am very grateful to Dr. Daniel Sakyi at the Department of Economics, KNUST - Kumasi, Ghana, and the anonymous reviewers for very insightful comments. Samuel Adjei, Alex Brobbey, and members of the data collection team are gratefully acknowledged. All errors are mine.

\section{Ethical issues}

No institutional approval was taken since the study did not involve clinical trials on participants. Further, we did not collect data from hospitals and no patient data or his/her disease episode was included. There was no risk to participants during or after the the study. In this case, obtaining approval from a specific institution was considered inapplicable.

Competing interests

Author declares that he has no competing interests.
Author's contribution

MKB is the single author of the manuscript.

\section{Endnotes}

[1] The formula is given by $n=\frac{N}{1+N(e)^{2}}$, where $n$ is the required sample size, $N$ is the population, and $e$ is the error level. The study assumed $95 \%$ confidence level.

${ }^{[2]}$ For the purposes of this study, all metropolitan, municipal, and district administrative areas are termed as districts.

${ }^{[3]}$ In the present study, a house means a dwelling unit or dwelling place for people. The residents may or may not share living arrangements. Thus, they may or may not eat from the same 'cooking pot.' Residents may or may not be blood relations. The house is selected so that eligible residents can be part of the study.

${ }^{[4]}$ See http://www.kwabre.ghanadistricts.gov.gh/ for details on communities in Kwabre East District.

\section{References}

1. Annemarie VW, Hester J. Patient choice of providers in a preferred provider organization. Medical Care. 1988;26:3:240255. doi:10.1097/00005650-198803000-00002

2. Appleby J, Harrison A, Devlin N. What is the Real Cost of More Patient Choice? London: The King's Fund; 2003. http://www. kingsfund.org.uk/publications/what-real-cost-more-patientchoice. Accessed March 25, 2014.

3. Ranerup A, Noren L, Sparud-Lundin C. Decision support systems for choosing a primary health care provider in Sweden. Patient Educ Couns. 2012;86(3):342-347. doi:10.1016/j. pec.2011.06.013

4. Dixon A, Robertson R, Bal R. The experience of implementing choice at point of referral: a comparison of the Netherlands and England. Health Econ Policy Law. 2010;5:295-317. doi:10.1017/ S1744133110000058

5. Sorensen AT. Insurer-hospital bargaining: negotiated discounts in post-deregulated Connecticut. J Ind Econ. 2003;51:469-490. doi:10.1111/j.0022-1821.2003.00211.x

6. Schwartz LM, Woloshin S, Birkmeyer JD. How do elderly patients decide where to go for major surgery? Telephone interview survey. BMJ. 2005;331(7520):821-824. doi:10.1136/ bmj.38614.449016.de

7. Montefiori M. Spatial competition for quality in the market for hospital care. Eur J Health Econ. 2005;6:131-135. doi:10.1007/ s10198-004-0268-8

8. Varkevisser M, van der Geest SA. Why do patients bypass the nearest hospital? An empirical analysis for orthopaedic care and neurosurgery in the Netherlands. Eur J Health Econ. 2007; 8(3):287-295. doi:10.1007/s10198-006-0035-0

9. Varkevisser M, van der Geest SA, Schut FT. Assessing hospital competition when prices don't matter to patients: the use of timeelasticities. Int J Health Care Finance Econ. 2010;10(1):43-60. doi:10.1007/s10754-009-9070-6

10. Atinga AR, Mensah SA, Asenso-Boadi F, Andoh-Adjei F. Migrating from user fees to social health insurance: exploring the prospects and challenges for hospital management. BMC Health Serv Res 2012;12:174. doi:10.1186/1472-6963-12-174

11. Boachie MK. Effects of Capitation on the Health Outcomes of Malaria Patients: Evidence from Ashanti and Brong Ahafo Regions of Ghana [MPhil (Economics) Thesis]. Kumasi, Ghana: Department of Economics, Kwame Nkrumah University of Sci \& Tech; 2013.

12. Dodoo JN. A stakeholder analysis of the capitation pilot under Ghana's National Health Insurance Scheme in the Ashanti Region [MPH Thesis]. Ghana: School of Public Health, University of Ghana; 2013.

13. Opoku M, Nsiah R, Oppong PA, Tetteh E. The effect of capitation payment on the national health insurance scheme in Ashanti 
Region, Ghana. School of Business, Kwame Nkrumah University of Science and Technology, Kumasi; 2014.

14. Rapid Appraisal of Capitation Payment in Ashanti Region: Kumasi. Ghana Health Service; [n.d].

15. Hirschman AO. Exit, Voice, and Loyalty: Responses to Decline in Firms, Organizations, and States. Cambridge, MA: Harvard University Press; 1970.

16. Naasegnibe K, Dary SK. Choice of healthcare providers among insured persons in Ghana. Research on Humanities and Social Sciences. 2012;2(10):88-97.

17. Dzator J, Asafu-Adjaye J. A study of malaria care provider choice in Ghana. Health Policy. 2004;69(3):389-401.

18. Amaghionyeodiwe LA. Determinants of the choice of healthcare provider in Nigeria. Health Care Manage Sci. 2008;11:215-227. doi:10.1007/s10729-007-9038-3

19. Adaman F, Ardiç OP, Erus B, Tüzemen D. Hospital choice: survey evidence from Istanbul. Turkish Studies. 2009;10(3):443468. doi:10.1080/14683840903141780

20. Bour D. Determinants of utilisation of health services by women in rural and urban areas in Ghana. GeoJournal. 2004;61:89-102. doi:10.1007/s10708-005-1929-6

21. White WD, Morrisey MA. Are patients travelling further? Int $J$ Econ Bus. 1998; 5:203-221.

22. Tai WC, Porell FW, Adams EK. Hospital choice of rural Medicare beneficiaries: patient, hospital attributes, and the patientphysician relationship. Health Serv Res. 2004;39:1903-1922.

23. Sofaer S, Crofton C, Goldstein E, Hoy E, Crabb J. What do consumers want to know about the quality of care in hospitals? Health Serv Res. 2005;40:2018-2036. doi:10.1111/j.14756773.2004.00324.x

24. Chul-Young R, Keon-Hyung L. Hospital choice by rural Medicare beneficiaries: does hospital ownership matter? A Colorado case. J Health Hum Serv Adm. 2005; 28(3):346-365.

25. 2010 Census Final Report. Ghana Statistical Service; 2012.

26. NHIA Annual Report. Accra; 2012.

27. Yamane T. Statistics: An Introductory Analysis. 2nd ed. New York: Harper and Row' 1967. 